\title{
Ladungsträgerbasierte Modellierung von Teilentladungsprozessen in Runddrahtwicklungen umrichtergespeister Niederspannungsmaschinen
}

\author{
N. Driendl@D, C. lütke Jüdefeld, F. Pauli, M. Schröder, K. Hameyer
}

\begin{abstract}
Das Auftreten von Teilentladungen in elektrischen Maschinen kann innerhalb von kurzer Zeit zum Versagen des Isoliersystems und somit zum Ausfall der Maschine führen. Für den Auslegungsprozess ist es daher essenziell zu ermitteln, ab welcher Spannung für welche Betriebspunkte Teilentladungen auftreten können. Umrichterbetriebene elektrische Maschinen sind aufgrund der transienten Spannungen besonderen Belastungen ausgesetzt. Insbesondere im Automobilbereich entstehen zusätzliche Anforderungen durch die Erhöhung der Zwischenkreisspannung und der Verwendung von schnellschaltenden Halbleiterelementen, wie z. B. Siliziumkarbid (SiC). Teilentladungen treten in luftgefüllten Hohlräumen innerhalb des Isoliersystems auf. Um die Teilentladungseinsetzspannung in Abhängigkeit von Spannungsanstiegsgeschwindigkeit und Überschwingverhalten zu berechnen, wird ein Modell auf Basis von hydrodynamischen Gleichungen entwickelt, das die Teilchenbewegung von positiven und negativen lonen sowie von Elektronen im Luftraum berücksichtigt. Mithilfe der ladungsträgerbasierten Modellierung ist es ferner möglich, den entstehenden Teilchenstrom zu berechnen, der einen Rückschluss auf die Teilentladungsintensität zulässt.
\end{abstract}

Schlüsselwörter: elektrische Maschine; Teilentladungen; Isoliersystem; SiC-Umrichter

\section{Charge carrier-based modeling of partial discharge processes in round wire windings of inverter-fed low-voltage machines.}

\begin{abstract}
The occurrence of partial discharges (PDs) in electrical machines can quickly lead to the failure of the insulation system and thus to the failure of the machine. For the design process, it is therefore essential to determine the voltage threshold for PD depending on the operating point. Inverter-driven electrical machines are exposed to distinctive loads due to the transient voltages. Particularly in the automotive sector, additional requirements arise due to the increase in the dc-link voltage and the application of fast-switching semiconductor materials, such as silicon carbide. Partial discharges occur in air-filled cavities within the insulation system. In order to calculate the partial discharge inception voltage (PDIV) subject to the voltage rise time and overshoot behavior, a model based on hydrodynamic equations is developed, which considers the diffusion of positive and negative ions as well as electrons in the air-filled discharge region. With the charge carrier-based modeling, it is possible to calculate the resulting current, which allows to consider the partial discharge intensity.
\end{abstract}

Keywords: electrical machine; partial discharge; insulation system; SiC inverter

Eingegangen am 16. Dezember 2020, angenommen am 16. Februar 2021, online publiziert am 4. März 2021 () The Author(s) 2021

\section{Einleitung}

Teilentladungen (TE) sind lokale Entladungen, die partiell die Isolationsstrecke zwischen zwei Elektroden überbrücken. Innerhalb des Statorisoliersystems einer Niederspannungsmaschine treten TE vorwiegend in gasgefüllten Teilvolumina im Bereich der Windungsisolierung auf. Entscheidend für das Entstehen einer Gasentladung ist das Überschreiten der kritischen Feldstärke, oberhalb derer die Isolierfähigkeit des Gases nicht mehr gegeben ist [1]. Dies kann im Betrieb der Maschine z. B. durch umrichterbedingte Überspannungen oder Temperatur und Druckveränderungen hervorgerufen werden [2, 3]. Insbesondere im Automotive-Bereich entstehen durch aktuelle technische Trends neue Anforderungen an das Isoliersystem. Bisher wurde in automobilen Traktionsanwendungen eine Zwischenkreisspannung im Bereich von $400 \mathrm{~V}$ verwendet. Um die Ladezeiten für die Batterie zu verkürzen, steht eine Erhöhung der Zwischenkreisspan- nung auf $800 \mathrm{~V}$ bevor [4]. Darüber hinaus bietet die Verwendung von schnell schaltenden Halbleitern die Vorteile von höheren Schaltfrequenzen und geringeren Schaltverlusten. Die hohe Spannungsanstiegsgeschwindigkeit regt jedoch auch hochfrequente Schwingungen an, die zu einem stärkeren Überschwingverhalten an den Maschinenklemmen führen. Das führt dazu, dass höhere Potentialdifferenzen zwischen den einzelnen Leitern entstehen und somit die Gefahr von Teilentladungen erhöht wird [5-8]. Die Windungsisolierung ist in der Regel die schwächste Komponente innerhalb

Driendl, Niklas, Institut für Elektrische Maschinen (IEM), RWTH Aachen University, Aachen, Deutschland (E-Mail: niklas.driendl@iem.rwth-aachen.de); lütke Jüdefeld, Clara, Institut für Elektrische Maschinen (IEM), RWTH Aachen University, Aachen, Deutschland; Pauli, Florian, Institut für Elektrische Maschinen (IEM), RWTH Aachen University, Aachen, Deutschland; Schröder, Michael, Institut für Elektrische Maschinen (IEM), RWTH Aachen University, Aachen, Deutschland; Hameyer, Kay, Institut für Elektrische Maschinen (IEM), RWTH Aachen University, Aachen, Deutschland 
des Isoliersystems, weshalb sich die Betrachtungen oftmals auf eine einfache Leiter-Leiter-Anordnung beschränken, um das Worst-CaseSzenario zu bewerten [9].

Obwohl eine einzelne Teilentladung nicht unmittelbar zu einem elektrischen Durchschlag führt, wird das Isoliersystem durch die Teilentladungen geschädigt. Mit dem Entladeprozess kommt es zu chemischen Prozessen, die die Isoliermaterialien altern lassen und die Lebensdauer der Isolation verkürzen. Bei herkömmlich isolierten Runddrahtwicklungen führt das Auftreten von TE bereits nach kurzer Wirkdauer zum Versagen der Isolierung und zum Ausfall der Maschine [1, 2]. Für eine Abschätzung der Belastbarkeit des Isoliersystems ist die Bestimmung der Teilentladungseinsetzspannung (engl.: Partial Discharge Inception Voltage, kurz: PDIV) unabdingbar. Dies kann sowohl simulativ als auch messtechnisch erfolgen.

In bekannten Modellen aus der Literatur $[10,11]$ wird oftmals die auf der Townsend-Gleichung basierende Paschenkurve verwendet, um die PDIV zu ermitteln. Bei diesen Modellen wird zunächst eine elektrostatische FE-Simulation durchgeführt, um für die betrachtete Anordnung das elektrische Feld zu ermitteln. Anschließend wird die Feldlösung mit der Paschenkurve, die die Durchschlagfestigkeit von elektrischen Gasen angibt, verglichen. Liegt der Wert für das elektrische Feld oberhalb der Paschenkurve, ist die kritische Spannung überschritten und TE können auftreten. Diese mathematische Beschreibung zur Isolationsfestigkeit ist jedoch nur für homogene bis schwach inhomogene, raumladungsfreie Felder zulässig [12]. In der betrachteten Anordnung treten jedoch Flächenladungen, die vom transienten Verlauf der Spannung abhängig sind, an den dielektrischen Barrieren auf. Somit reicht die beschriebene Modellierung mit der Paschenkurve nicht aus.

In dieser Arbeit wird ein Ansatz zur Berechnung der PDIV vorgestellt, der basierend auf hydrodynamischen Gleichungen die Ladungsträgerbewegungen innerhalb der Anordnung berücksichtigt. Mithilfe dieses Ansatzes ist es möglich, neben der Ansammlung von Flächenladungen auch die Stromstärke zu berechnen, welche bei einer Entladung entsteht. Dadurch können Rückschlüsse auf die Intensität der Entladung gezogen werden. In Abschn. 2 wird der Modellierungsansatz erläutert. Anschließend wird in Abschn. 3 der Versuchsaufbau beschrieben, der zur Validierung des Modells verwendet wird. Im darauffolgenden Abschnitt werden Mess- und Simulationsergebnisse diskutiert und verglichen. Abschließend folgt eine Zusammenfassung der Arbeit.

\section{Modellierung von Teilentladungsprozessen in elektrischen Niederspannungsmaschinen}

Zur Modellierung von Teilentladungsprozessen innerhalb der Statorisolierung einer Niederspannungsmaschine mit Runddrahtwicklungen wird eine zweidimensionale Ersatzanordnung eingeführt und die Entladung als Gasentladung charakterisiert. In Analogie zur Geometrie verdrillter Prüflinge nach [13] wird die Entladeanordnung auf zwei parallele Runddrahtleiter in Luft vereinfacht [3]. Abbildung 1 veranschaulicht die Ersatzanordnung, die hauptsächlich durch den Leiterradius $r_{\text {Leiter }}$ und die Lackschichtdicke der Isolation $d_{\text {Iso }}$ definiert ist. Durch eine elektrostatische Finite-Elemente-Berechnung kann die spannungsabhängige Feldverteilung in der Luft bestimmt werden, welche Ausgangspunkt der Modellierung eines Gasentladeprozesses ist.

\subsection{Entladeprozesse in Gasen}

Bei einem Gasentladeprozess steigt die Leitfähigkeit eines Gasvolumens, sodass sich die Elektroden über die Gasstrecke entladen können. Die Leitfähigkeit eines Gases wird durch Stoß- und Photoionisationen erhöht, bei denen neutrale Gasmoleküle zu negativen

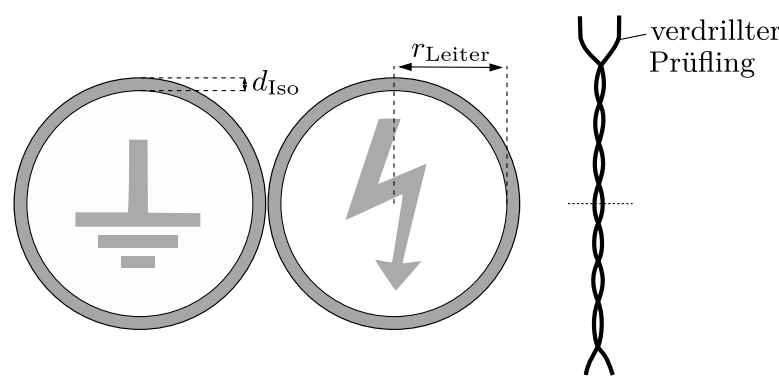

Abb. 1. Ersatzanordnung als Querschnitt von zwei verdrillten Lackdrahtleitern

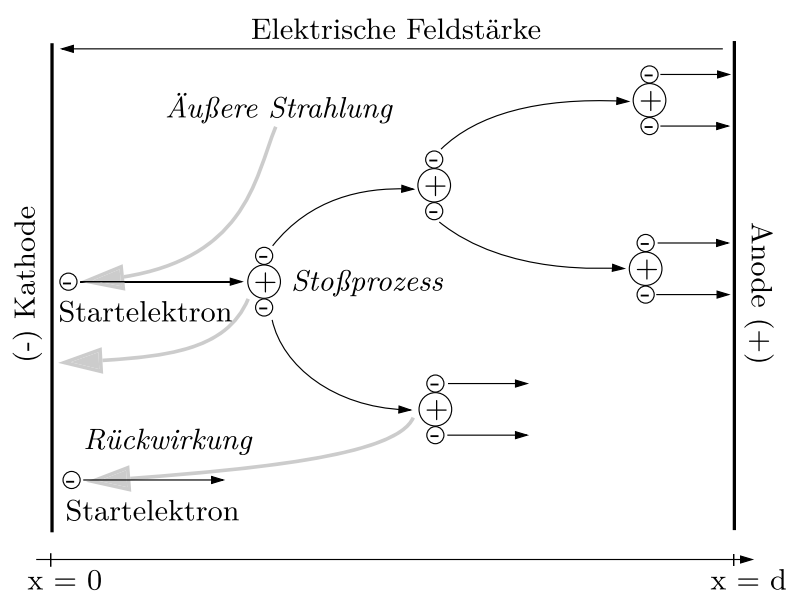

Abb. 2. Schematische Darstellung des Generationsmechanismus nach Townsend [12]

und positiven Ladungsträgern aufgespalten werden. Diese Prozesse finden sowohl im Gas als auch an den Elektrodenoberflächen statt. Nach dem Generationsmechanismus nach Townsend kann jedes freie Elektron im Gas einen Entladeprozess auslösen. Bei ausreichender Feldstärke ionisiert dieses Elektron während der Beschleunigung in Richtung Anode weitere Gasmoleküle und es kommt zu einem lawinenartigen Wachstum an freien Ladungsträgern. Abbildung 2 veranschaulicht diesen Prozess. Die dabei entstandenen positiven lonen bewegen sich in Richtung Kathode. An der Kathodenoberfläche können Sekundärelektronen herausgelöst werden. Diese können sowohl durch den Aufprall positiver lonen als auch durch Photonen entstehen. Sekundärelektronen stoßen als Startelektronen auf dem Weg zur Anode ebenfalls Folgelawinen an. Dieser Vorgang wird Generationsmechanismus genannt. Sobald von jeder Lawine mindestens eine weitere Lawine ausgelöst wird, steigt die Anzahl der freien Ladungsträger im Gasraum und die Townsend'sche Zündbedingung ist erfüllt [12]:

$$
\gamma \cdot\left[\exp \left(\int_{x=0}^{d} \alpha \cdot d x\right)-1\right] \geq 1 .
$$

Die mathematische Formulierung der Townsend'schen Zündbedingung für eine inhomogene Feldverteilung ist durch den Zusammenhang in (1) gegeben. Der lonisierungskoeffizient $\alpha$ gibt die mittlere Anzahl der lonisierungen an, die ein Elektron entlang einer Strecke hervorruft. Der Sekundärkoeffizient beschreibt die Wahrscheinlichkeit für das Herauslösen eines Sekundärelektrons je auftreffendem Ion oder Photon. Durch Multiplikation des Sekundärkoeffizienten $\gamma$ mit der je Lawine netto erzeugten Elektronenanzahl 


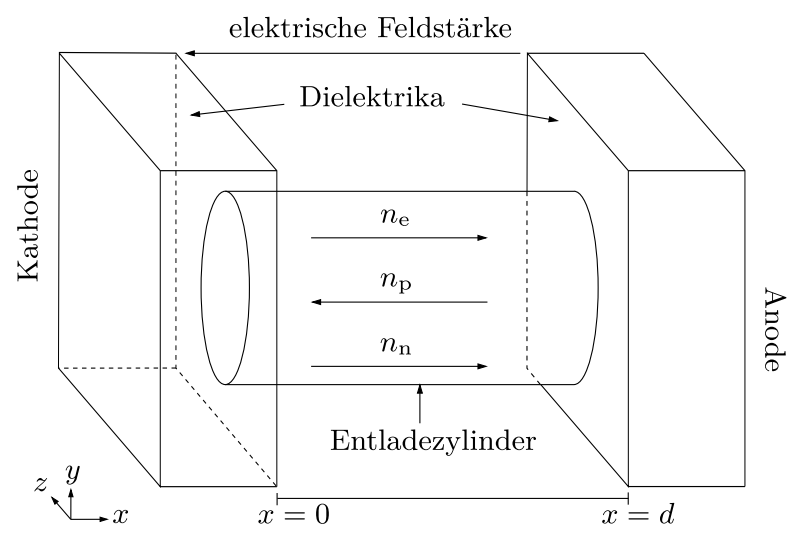

Abb. 3. Zylinderförmiger Entladekanal in homogener Anordnung mit zwei dielektrischen Barrieren

errechnet sich die Anzahl an neuen Startelektronen an der Kathode. Anode und Kathode befinden sich analog zur Abb. 2 bei $x=d$ und $x=0$. In Anwendung auf die Feldverteilung der Ersatzanordnung für die Windungsisolierung einer Niederspannungsmaschine lässt sich mit dem Townsend-Kriterium die Teilentladungseinsetzspannung des Statorisoliersystems abschätzen. Dabei wird die Spannung berechnet, ab der die Bedingung in (1) entlang mindestens einer Feldlinie im Gasraum der Ersatzanordnung erfüllt ist. Die Abschätzung der Teilentladungseinsetzspannung mit dem TownsendKriterium erlaubt ausschließlich statische Betrachtungen des Entladegeschehens, da sowohl transiente Spannungsverläufe als auch die Ansammlung von Ladungsträgern nicht berücksichtigt werden können.

\subsection{Teilentladungsmodell nach dem Plasma-Ansatz}

Eine umfassendere Modellbildung erfolgt nach dem Ansatz der Plasmamodelle. Der Plasma-Ansatz beruht auf der Beschreibung der Ladungsbewegungen durch ein System aus Fluss-Kontinuitätsgleichungen jeder Ladungsträgerart. Die Ladungsbewegung in der gasgefüllten Entladestrecke steht im direkten Zusammenhang mit dem Entladestrom und wird zeit-diskret berechnet. Ursprünglich wurde dieser Ansatz entwickelt, um Entladungen zwischen metallischen Elektroden mit elektrischer Spannungsdifferenz zu modellieren [14]. Unter Berücksichtigung der sich an den Barrieren ansammelnden Flächenladung und deren Einfluss auf die Feldverteilung kann dieser Ansatz jedoch auch zur Modellierung von Gasentladungen zwischen dielektrisch beschichteten Elektroden (Barriereentladung) verwendet werden.

Optische Untersuchungen von dielektrischen Barriereentladungen aus der Literatur weisen darauf hin, dass sich Teilentladungen im Gasraum zwischen isolierten Elektroden in Form einzelner räumlich abgegrenzter Entladekanäle ausbilden. Es wird die vereinfachende Annahme getroffen, dass die Dichten der geladenen Teilchen und die elektrische Feldstärke über dem Querschnitt des zylinderförmigen Entladekanals konstant sind. Die elektrische Feldstärke entspricht dabei dem Wert auf der Kanalachse [15]. Abbildung 3 zeigt die schematische Darstellung eines Entladekanals zwischen zwei isolierten Platten mit konstanter Spannungsdifferenz. Die Achse des Kanals ist parallel zur x-Achse, sodass die Teilchendichten und die elektrischen Felder nur eine Abhängigkeit von der x-Koordinate besitzen. Unter Anwendung der sogenannten LocalField-Approximation (LFA) lässt sich für jede zeit- und raumabhängige Teilchendichte $n_{i}$ mit der Driftgeschwindigkeit $v_{i}$, der Diffusions- konstanten $D_{i}$ und dem Quellterm $S_{i}$ die allgemeine Kontinuitätsgleichung formulieren [19]:

$$
\frac{\partial n_{i}}{\partial t}+\nabla\left(n_{i} v_{i}-D_{i} \nabla n_{i}\right)=S_{i}
$$

Die Variable $i$ steht als Platzhalter für die verschiedenen Ladungsträgerarten. Die Variablen $x$ und $t$ bezeichnen die Ortskoordinate bzw. den Zeitpunkt. Die auf eine Dimension reduzierten Kontinuitätsgleichungen für Elektronen $e$, positive Ionen $p$ und negative Ionen $n$ mit eingesetzten Quelltermen sind in den Gleichungen (3) bis (5) zusammengefasst. Auf den Diffusionsterm wird dabei verzichtet, da er einen geringen Einfluss auf das Berechnungsergebnis hat [14].

$$
\begin{aligned}
\frac{\partial n_{\mathrm{e}}}{\partial t} & =S_{\mathrm{e}}+n_{\mathrm{e}} \alpha\left|v_{\mathrm{e}}\right|-n_{\mathrm{e}} \eta\left|v_{\mathrm{e}}\right|-n_{\mathrm{e}} n_{\mathrm{p}} \beta-\frac{\partial\left(n_{\mathrm{e}} v_{\mathrm{e}}\right)}{\partial x} \\
\frac{\partial n_{\mathrm{p}}}{\partial t} & =n_{\mathrm{e}} \alpha\left|v_{\mathrm{e}}\right|-n_{\mathrm{e}} n_{\mathrm{p}} \beta-n_{\mathrm{n}} n_{\mathrm{p}} \beta-\frac{\partial\left(n_{\mathrm{p}} v_{\mathrm{p}}\right)}{\partial x} \\
\frac{\partial n_{\mathrm{n}}}{\partial t} & =n_{\mathrm{e}} \eta\left|v_{\mathrm{e}}\right|-n_{\mathrm{n}} n_{\mathrm{p}} \beta-\frac{\partial\left(n_{\mathrm{n}} v_{\mathrm{n}}\right)}{\partial x}
\end{aligned}
$$

Die Bewegungs- und Generationsprozesse sind vorwiegend durch die sogenannten Schwarmparameter bestimmt. Sie umfassen die Driftgeschwindigkeiten der Elektronen, positiven lonen und negativen lonen $v_{\mathrm{e}}, v_{\mathrm{p}}$ und $v_{\mathrm{n}}$ und die Koeffizienten für lonisation, Anlagerung, und Rekombination $\alpha, \eta$ und $\beta$. Der Quellterm $S_{\mathrm{e}}$ beinhaltet alle zusätzlichen Generationsprozesse von Elektronen, die nicht durch Stoßprozesse im Gas bedingt sind, wie z. B. die Sekundäremission.

Die Kontinuitätsgleichungen stellen einen Zusammenhang zwischen zeitlicher Änderung der Teilchendichte, Divergenz der Strömung und Generationsprozessen her. Mithilfe eines Fluss-Korrigierten-Transportalgorithmus nach [16, 17] kann das Differentialgleichungssystem iterativ gelöst werden und die zeit- und raumabhängigen Ladungsträgerdichten bestimmt werden. Die Schwarmparameter sind abhängig von der lokalen reduzierten Feldstärke $E / n$, die dem Quotienten aus elektrischer Feldstärke $E$ und neutraler Teilchendichte $n$ entspricht. Da sich die Ladungsträger nicht ungehindert durch die dielektrische Schicht an Kathode und Anode bewegen können, müssen Ladungsträgeransammlungen berücksichtigt werden. An der Kathode sammeln sich positive lonen und an der Anode Elektronen in Form einer Oberflächenladung an. Wie in den Gleichungen (6) und (7) formuliert, entspricht die zeitliche Änderung der Flächenladungsdichte $\sigma$ dem Teilchenfluss an der Grenze zwischen Gas und Isolierung multipliziert mit der Elementarladung e [18]. Die Grenzen des Gasraums befinden sich bei $x=0$ und $x=d$.

$$
\begin{aligned}
& \frac{\partial \sigma_{\text {Kathode }}}{\partial t}=e \cdot n_{\mathrm{p}}(0, t) \cdot v_{\mathrm{p}}(0, t) \\
& \left.\frac{\partial \sigma_{\text {Anode }}}{\partial t}=e \cdot\left[n_{\mathrm{e}}(d, t) \cdot v_{\mathrm{e}}(d, t)+n_{\mathrm{n}}(d, t) \cdot n_{\mathrm{e}}(d, t)\right)\right]
\end{aligned}
$$

Bei der Modellierung des Elektronenquellterms $S_{e}$ werden mehrere Mechanismen an der Oberfläche des Kathodenmaterials berücksichtigt. Abbildung 4 veranschaulicht die unterschiedlichen Prozesse, auf welche im Folgenden näher eingegangen wird.

Grundsätzlich wird davon ausgegangen, dass die Flächenladungen ohne feldbedingte Krafteinwirkung im Zeitbereich einiger Millisekunden unverändert auf den Oberflächen verbleiben [19]. Wechselt die Spannung jedoch das Vorzeichen, sodass die Kathode eine negative Flächenladung aufweist, können dort angelagerte Elektronen abgelöst werden und freie Startelektronen bilden. Dieser Prozess wird im Teilentladungsmodell durch thermische Adsorption berücksichtigt, bei der sich die Elektronen mit einer temperaturabhängigen Desorptionsfrequenz $f_{\text {Des }}(T)$ ablösen [20]. Der momentane 


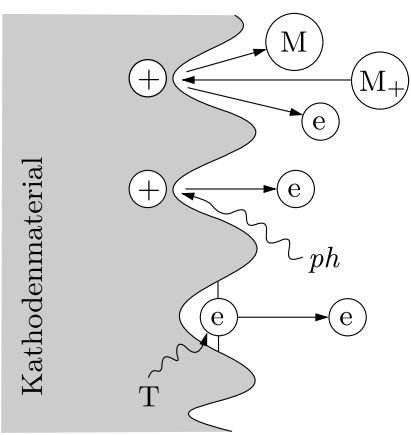

Sekundäremission durch positive Ionen

Sekundäremission durch Photoemission

Thermische Desorption

Abb. 4. Veranschaulichung der verschiedenen Generationsprozesse an der Kathodenoberfläche [20]

Elektronenfluss $n_{\mathrm{e}}^{\text {Des }}(0, t) \cdot v_{\mathrm{e}}(0, t)$ unmittelbar an der Kathodenbarriere ergibt sich durch Desorption zu Gleichung (8). Die Desorptionsfrequenz berechnet sich nach [20] in Abhängigkeit der Adsorptionsenergie $\Phi_{\sigma}$, welche im Bereich von $1 \mathrm{eV}$ liegt, und der Fluchtfrequenz der angesammelten Elektronen $f_{\text {esc }}$.

$$
n_{\mathrm{e}}^{\text {Des }}(0, t) \cdot v_{\mathrm{e}}(0, t)=\frac{\sigma_{\text {Kathode }} \cdot f_{\text {Des }}}{e}
$$

Neben den Ionisations- und Anlagerungsprozessen im Gas werden ebenfalls die Generationsprozesse an der Kathodenoberfläche modelliert. Bei den betrachteten Primärteilchen, die Sekundärelektronen herauslösen, handelt es sich ausschließlich um positive Ionen oder Photonen. Der Sekundärelektronenfluss entspricht dabei jeweils dem momentanen Primärteilchenfluss an der Kathode multipliziert mit dem Sekundärkoeffizienten $\gamma$. Dividiert durch die Elektronengeschwindigkeit $v_{\mathrm{e}}(0, t)$ an der Kathode ergibt sich die entsprechende Elektronendichte $n_{\mathrm{e}}(0, t)$. Für positive Ionen formuliert sich die Sekundärelektronendichte an der Kathode $n_{\mathrm{e}}^{\text {lon }}$ unter Berücksichtigung des spezifischen Sekundärkoeffizienten そıon zu Gleichung (9) [19]:

$$
n_{\mathrm{e}}^{\text {lon }}=\gamma_{\text {lon }} \frac{n_{\mathrm{p}}(0, t) \cdot v_{\mathrm{p}}(0, t)}{v_{\mathrm{e}}(0, t)}
$$

Die durch auftreffende Photonen herausgelösten Elektronen werden mit Gleichung (10) berechnet [21].

$$
n_{\mathrm{e}}^{\mathrm{Ph}}=\frac{\gamma_{\mathrm{Ph}}}{v_{\mathrm{e}}(0, t)} \int_{0}^{d} \alpha(x, t) \cdot n_{\mathrm{e}}(x, t) \cdot v_{\mathrm{e}}(x, t) \cdot \Omega(x) \mathrm{d} x
$$

Der Photonenfluss an der Kathode ist abhängig von der lonisationsrate $\alpha \cdot n_{\mathrm{e}} \cdot v_{\mathrm{e}}$. Integriert über den gesamten Entladekanal ergibt sich der gesamte momentane Photonenfluss der Einzelentladung. Mit dem geometrischen Faktor $\Omega(x)$ wird berücksichtigt, dass sich nicht alle Photonen in Richtung der Kathode bewegen.

Die Raumladungsdichte an einem Ort $x$ zum Zeitpunkt $t$ im Entladekanal entspricht der Netto-Ladungsträgerdichte multipliziert mit der Elementarladung.

$$
\rho(x, t)=e \cdot\left(n_{\mathrm{p}}(x, t)-n_{\mathrm{e}}(x, t)-n_{\mathrm{n}}(x, t)\right)
$$

Sowohl die angesammelte Flächenladung als auch die Raumladung im Entladekanal beeinflussen die lokale elektrische Feldstärke und damit die Schwarmparameter. Dynamisch werden die Feldbeiträge der Flächen- und Raumladungen bestimmt und in der lokalen reduzierten Feldstärke entlang des Entladekanals berücksichtigt. Durch die vereinfachende Annahme, dass sich die Flächenladungen homogen im Radius des Entladekanals auf den dielektrischen Oberflächen

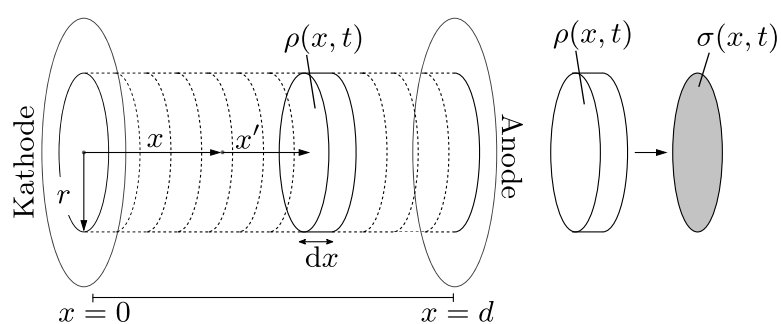

Abb. 5. Unterteilung des Entladekanals in einzelne Scheiben für die numerische Berechnung des Raumladungsfeldes [18]

verteilen, kann das Feld aus Flächenladungen analog zum Feld einer geladenen Scheibe analytisch bestimmt werden. Das Raumladungsfeld auf der Entladekanalachse wird nach dem selben Ansatz mit der sogenannten Scheiben-Methode berechnet. Abbildung 5 verdeutlicht die Unterteilung des Entladekanals in Scheiben mit dem Kanalradius $r$ und endlicher Dicke $\mathrm{d} x$. Es werden die Feldbeiträge infinitesimal dünner Schreiben mit gleichen homogen verteilten Ladungsmengen bestimmt. Durch Superposition der Feldbeiträge aller Scheiben an den Raumpunkten $x^{\prime}$ kann das Feld $E_{\rho}$ an einem Punkt $x$ auf der Kanalachse durch Gleichung (12) berechnet werden [18].

$$
\begin{aligned}
& E_{\rho}(x, t)=\frac{1}{2 \varepsilon_{0}}\left\{\int_{-x}^{0} \rho\left(x+x^{\prime}, t\right)\left[-1-x^{\prime}\left(x^{\prime 2}+r^{2}\right)^{-\frac{1}{2}}\right] \mathrm{d} x^{\prime}\right. \\
& \left.+\int_{0}^{d-x} \rho\left(x+x^{\prime}, t\right)\left[1-x^{\prime}\left(x^{\prime 2}+r^{2}\right)^{-\frac{1}{2}}\right] \mathrm{d} x^{\prime}\right\}
\end{aligned}
$$

Während der gesamten Entladung muss das Integral über die elektrische Feldstärke von der metallischen Anode zur metallischen Kathode der Elektrodenspannung entsprechen. Bedingt durch die zwingende Konsistenz zwischen elektrischer Feldstärke und Spannung kann die Kompensationsladung $Q_{\text {Komp }}$ auf den metallischen Elektroden bestimmt werden. Durch die zeitliche Ableitung der Kompensationsladung kann die messbare Stromstärke /Mess bestimmt werden, die bedingt durch den Entladeprozess in die Anordnung hineinfließt.

$$
I_{\text {Mess }}=\frac{\mathrm{d} Q_{\mathrm{Komp}}}{\mathrm{d} t}
$$

Der Entladestrom, der innerhalb eines Entladekanals im Gasraum fließt, ist der sogenannten Sato-Strom. Dieser trägt maßgeblich zur chemischen Zersetzung der Isoliermaterialien bei. Der zeitabhängige Entladestrom errechnet sich nach Gleichung (14). Dabei wird die mittlere Ladungsträgerverschiebung aus Ionen und Elektronen bestimmt und mit der Elementarladung e und der Querschnittsfläche $\pi \cdot r^{2}$ des Entladekanals multipliziert. Je höher die Dichten der Ladungsträger $n_{i}$ und je größer die Geschwindigkeiten $v_{i}$, desto größer der Sato-Strom /sato. Der Gesamtentladestrom entspricht der Summe aller Strombeiträge der einzelnen Kanäle. Wie viele Entladekanäle in der Gesamtanordnung entstehen ist dabei geometrieabhängig.

$$
I_{\text {Sato }}=\frac{e \cdot \pi r^{2}}{d} \int_{0}^{d}\left(n_{\mathrm{p}} \cdot\left|v_{\mathrm{p}}\right|+n_{\mathrm{n}} \cdot\left|v_{\mathrm{n}}\right|+n_{\mathrm{e}} \cdot\left|v_{\mathrm{e}}\right|\right) \mathrm{d} x
$$

\subsection{Anwendung des Plasma-Ansatzes auf die \\ Ersatzanordnung}

Die Teilentladungs-Simulation für die Ersatzanordnung der Wicklungsisolierung einer Niederspannungsmaschine basiert auf der Berechnung des statischen Laplace-Feldes im Luftraum. Mit Kenntnis der materialabhängigen Sekundärkoeffizienten kann aus der Feldlösung unter Anwendung des Townsend-Kriteriums die kritische Feldlinie bestimmt werden, um welche sich der Entladekanal mit hoher 


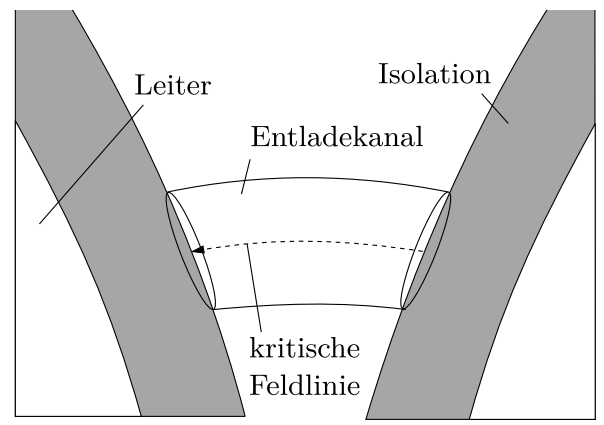

Abb. 6. Exemplarischer Entladekanal um die kritische Feldlinie innerhalb der Ersatzanordnung

Wahrscheinlichkeit ausbilden wird. Die kritische Feldlinie ist dadurch bestimmt, dass entlang ihres Verlaufs für die geringste Elektrodenspannung die Zündbedingung erfüllt ist. Die Entladekanallänge entspricht dann der Länge der Feldlinie. In Abb. 6 ist ein exemplarischer Entladekanal dargestellt. Die meist leichte Krümmung der Feldlinie wird begradigt, um die Feldberechnung mit der Scheiben-Methode anwenden zu können. Der Kanalradius $r$ kann durch einen experimentell bestimmten Zusammenhang aus der Literatur in Abhängigkeit der Kanallänge $d$ und der neutralen Teilchendichte $n$ nach Gleichung (15) bestimmt werden [21]

$$
r_{\text {Kanal }}=150 \cdot 10^{-6} \mathrm{~m} \cdot \sqrt{\frac{\sqrt{d}}{n} \cdot 26,5 \cdot 10^{24} \mathrm{~m}^{-3}}
$$

Mit Kenntnis der Kanalgeometrie und der Modellparameter kann der Teilentladungsprozess mithilfe des Plasma-Ansatzes für einen repräsentativen Entladekanal simuliert werden. Transiente Spannungsverläufe zwischen den Elektroden werden modelliert, indem die raum- und flächenladungsfreie Feldkomponente variiert wird. Um die Simulationsergebnisse mit Messergebnissen an verdrillten Prüflingen vergleichen zu können, muss der je Entladekanal messbare Strom, der sich durch die Konsistenz zwischen Elektrodenspannung und elektrischer Feldstärke ergibt, mit der Anzahl der Entladekanäle multipliziert werden. In der zweidimensionalen Betrachtung entsteht ober- und unterhalb des Berührungspunktes der Drähte jeweils nur ein Kanal. Es wird die vereinfachende Annahme getroffen, dass die Entladekanäle gleichmäßig entlang der Verdrillung auftreten und direkt aneinander angrenzen. Die Anzahl der Entladekanäle $N_{\text {Kanal }}$ ergibt sich analog zu Abb. 7 in Abhängigkeit der Länge der Verdrillung /verdrillt zu:

$$
N_{\text {Kanal }}=\frac{\text { Verdrillt }}{r_{\text {Kanal }}} .
$$

\section{Versuchsaufbau}

Die für die Windungsisolation kritischen Belastungen im Umrichterbetrieb der Maschine sind geprägt von schnellen, bipolaren Spannungsformen. Der schnelle Spannungsanstieg regt dabei hochfrequente Schwingungen an, die reflexionsbedingt zum Überschwingen der Spannungen an den Maschinenklemmen führen. Ein exemplarischer Überschwingvorgang ist in Abb. 8 dargestellt. Der Scheitelwert der Spannung ist durch $U_{p}$ gekennzeichnet und $U_{a}$ ist der eingeschwungene Spannungswert, welcher der Zwischenkreisspannung entspricht. Die Anstiegszeit ist durch den mit $t_{\mathrm{r}}$ markierten Zeitbereich gekennzeichnet. Dieser ist durch die Zeitpunkte $t_{10}$ und $t_{90}$ definiert, an denen $10 \%$ bzw. $90 \%$ des Spannungshubes $\left(U_{p}-\left(-U_{a}\right)\right)$ abgeschlossen sind.

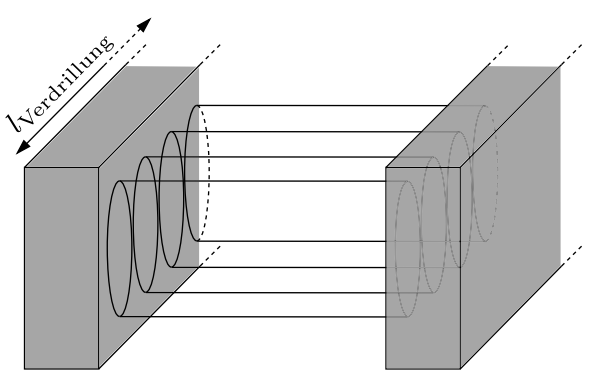

Abb. 7. Aufteilung der Entladekanäle auf die Länge der Verdrillung

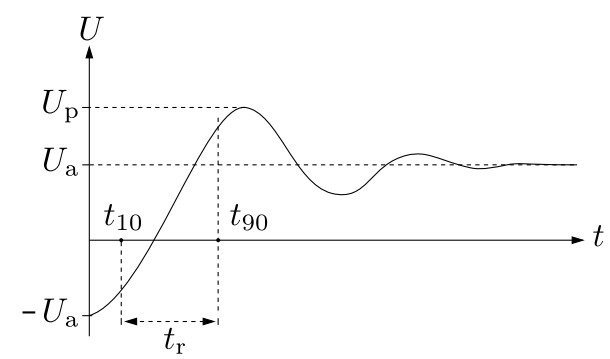

Abb. 8. Exemplarischer Spannungsverlauf eines Überschwingvorgangs analog zu [22]

Tab. 1. Charakteristische Größen der Prüfspannungen mit den IEMPulsgeneratoren

\begin{tabular}{ll}
\hline Parameter & Wertebereich \\
\hline $\begin{array}{l}\text { Spannungsform } \\
\text { Zwischenkreisspan- } \\
\text { nung }\end{array}$ & $\begin{array}{l}\text { Bipolare Rechteckspannung } \\
0 \mathrm{~V} . \ldots 1200 \mathrm{~V}\end{array}$ \\
$\begin{array}{l}\text { Anstiegszeit } \\
\text { Spannungsanstiegs- } \\
\text { geschwindigkeit }\end{array}$ & $40 \mathrm{~ns} . .120 \mathrm{~ns}$ \\
Frequenz & $10 \mathrm{kV} \mathrm{\mu s}{ }^{-1} \ldots 45 \mathrm{kV} \mathrm{\mu s}^{-1}$ \\
\hline
\end{tabular}

Um das Teilentladungsverhalten an verdrillten Prüflingen für möglichst ähnliche Beanspruchungen zu untersuchen, werden Hochvolt$\mathrm{SiC}-$ Pulsgeneratoren verwendet. Die am Institut für Elektrische Maschinen entwickelten Pulsgeneratoren werden in [23] vorgestellt. Diese generieren frequenz- und amplitudenvariable Rechteckspannungen mit schnellen Anstiegszeiten. Die charakteristischen Größen der Ausgangsspannung sind in Tab. 1 zusammengefasst.

Der untersuchte Runddraht Dahréntråd DAMID 200 der Firma LWW Group entspricht den Lieferbedingungen nach DIN EN 60317-0-1 [24] und ist ein Zweischicht-Lackdraht des Typs Grad 2. Die Grundlackschicht besteht aus THEIC-modifiziertem Polyester oder Polyesterimid (PEI), der Überzuglack aus Polyamidimid (PAI). Abbildungen 9 und 10 zeigen den zeitlichen Verlauf der Prüfspannung an den Klemmen eines Pulsgenerators und den schematischen Aufbau für die Teilentladungsmessung. Die verdrillten Prüflinge werden an den Klemmen des Pulsgenerators angeschlossen. Über das Signal einer Antenne werden die hochfrequenten Wellen der schnellen Gasentladungen empfangen und Teilentladungen detektiert. Parallel zur TE-Überwachung werden Spannung und Stromstärke am Prüfling mit einem Oszilloskop erfasst. Indem die Zwischenkreisspannung so lange erhöht wird, bis mit der Antenne TE detektiert werden, kann die Teilentladnungseinsetzspannung bestimmt werden. 


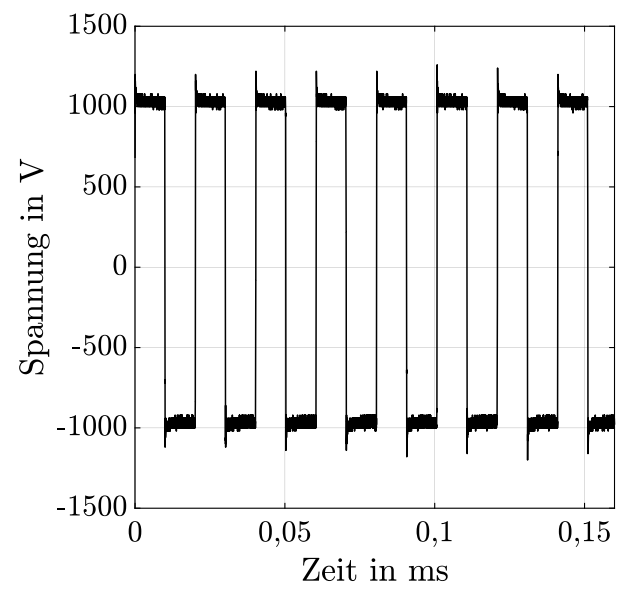

Abb. 9. Zeitlicher Verlauf der Rechteckspannungen bei $50 \mathrm{kHz}$

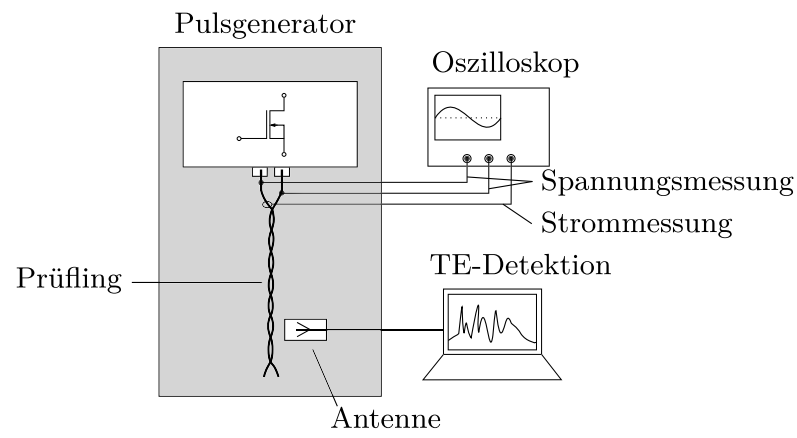

Abb. 10. Schematischer Aufbau des Teilentladungsprüfstands für bipolare Rechteckspannungen

\section{Simulations- und Messergebnisse}

Im Folgenden werden die Simulationsergebnisse vorgestellt. Zunächst wird auf die Berechnung der kritischen Feldlinie eingegangen, auf der die weiteren Berechnungsschritte basieren. Anschließend werden die berechneten zeitlichen Strom- und Spannungsverläufe sowie der Verlauf der Flächenladung der Simulation diskutiert. Es wird insbesondere der Unterschied zwischen unipolarer und bipolarer Impulsspannung untersucht. Die verwendeten Simulationsparameter sind in Tab. 2 im Anhang aufgelistet. Durch Vergleich mit Messergebnissen wird der Simulationsansatz validiert.

\subsection{Kritische Feldlinie}

Die kritische Feldlinie wird entsprechend der in Abschn. 2 vorgestellten Methodik berechnet. Dies bedeutet, dass das TownsendKriterium nach (1) für genau eine Feldlinie erfüllt ist. In Abb. 11 ist die berechnete Anzahl an Sekundärelektronen je Lawine für verschiedene Spannungen und Feldlinien zu sehen. Bei einer Spannung von $1040 \mathrm{~V}$ ist die berechnete Lawinenrückwirkung nur für eine Feldlinie größer gleich eins und somit das Townsend-Kriterium erfüllt.

In Abb. 12 (a) sind die Feldlinienverläufe im Luftbereich exemplarisch dargestellt und die kritische Feldlinie hervorgehoben. Es wird deutlich, dass die kritische Feldlinie nicht im Bereich des geringsten Abstandes zwischen den Leitern zu erwarten ist, sondern in etwa 1,7 mm oberhalb des Berührpunktes. Die Anordnung ist spiegelsymmetrisch zur x-Achse, somit gibt es immer zwei kritische Feldlinien. Wird die Spannung der Anordnung weiter erhöht, vergrößert sich entlang der Oberfläche der Isolierung der Bereich, an dem die

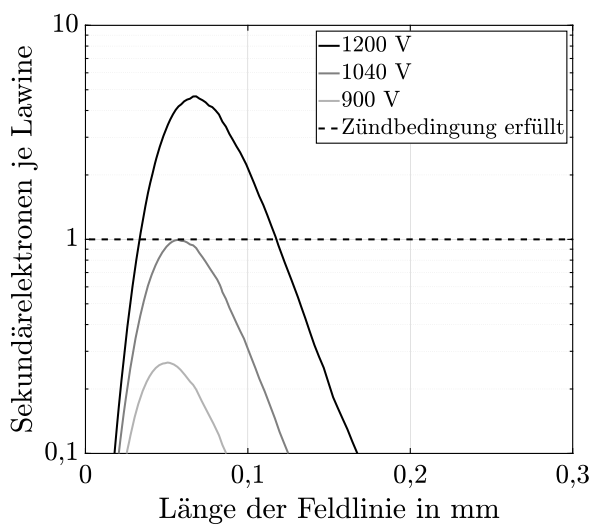

Abb. 11. Auswertung des Townsend-Kriteriums für verschiedene Spannungen

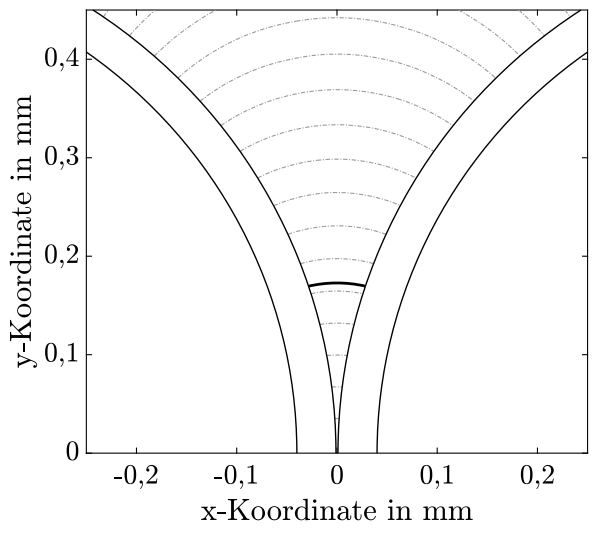

(a) Kritische Feldlinie.

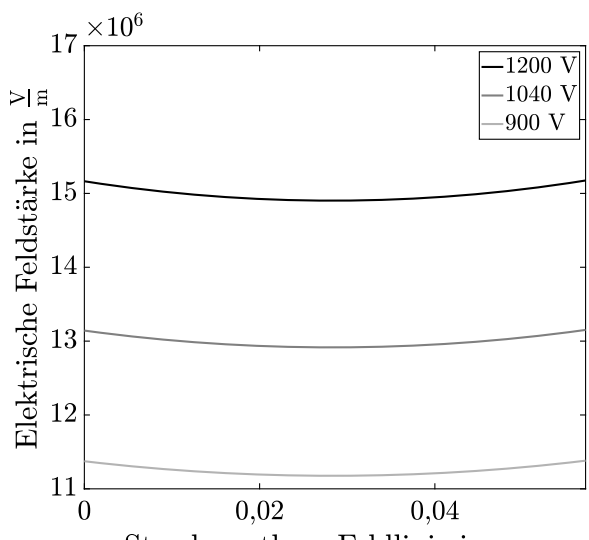

Strecke entlang Feldlinie in $\mathrm{mm}$

(b) Verlauf der elektrischen Feldstärke.

Abb. 12. Kritische Feldlinie zwischen den Drahtoberflächen und Verlauf der elektrischen Feldstärke entlang der kritischen Feldlinie

Zündbedingung erfüllt ist. Wie bereits in Abschn. 2.3 erläutert, ist durch die Begradigung der kritischen Feldlinie kein signifikanter Fehler zu erwarten, da diese nur einen schwach inhomogenen Verlauf aufweist, wie in Abb. 12 (b) zu sehen ist.

\subsection{Oberflächenladungen und Ladungsträgerströme} Für die Simulation des zeitlichen Verlaufs der Oberflächenladungen und Ladungsträgerströme werden die mit dem Oszilloskop 


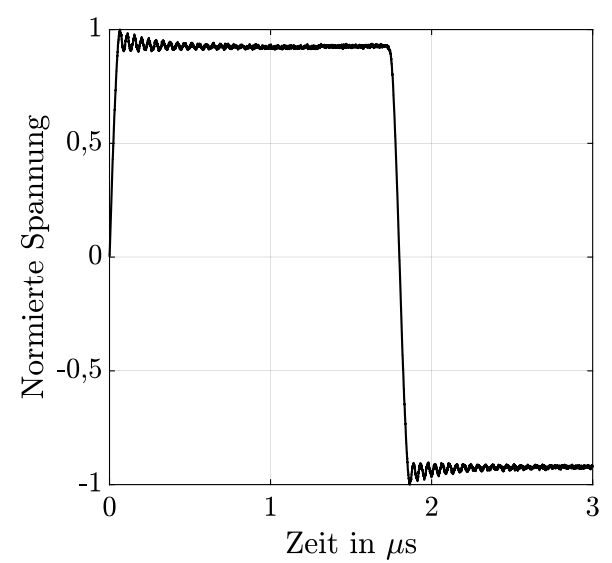

(a) Bipolare Impulsspannung.

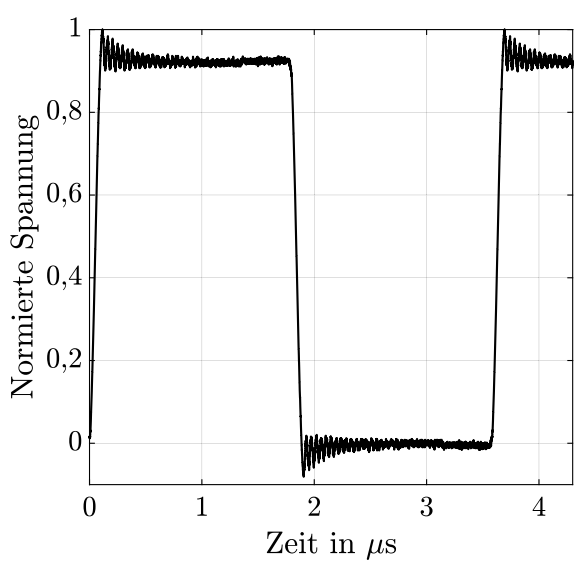

(b) Unipolare Impulsspannung.

Abb. 13. Auf den Scheitelwert normierte Spannungsverläufe für bipolare und unipolare Impulsspannungen

aufgenommenen Spannungsimpulse als Eingangsdaten verwendet. Die unipolare und bipolare Testspannung ist in normierter Form in Abb. 13 dargestellt. Die Testspannung wird in ihrer Amplitude skaliert. Der Überschwingfaktor beträgt für beide Spannungsformen circa 1,09 bei einer Spannungsanstiegszeit von etwa $80 \mathrm{~ns}$. Das entspricht einem du/dt von $30 \mathrm{kV} / \mu \mathrm{s}$.

Beobachtungen der Teilentladungs-Charakteristiken zeigen, dass Teilentladungen überwiegend an den steigenden und fallenden Flanken der Spannungspulse auftreten, sofern der Prüfkörper zuvor keinen Alterungsprozessen ausgesetzt wurde [25]. Zur Verringerung der Rechenzeit des Simulationsmodells wird die Pulsweite des Spannungspulses gegenüber der tatsächlichen gemessenen Spannung verringert. Der Berechnungszeitraum ist ausreichend, um für die Ladungsträgeransammlungen einen stationären Zustand zu erreichen.

\subsubsection{Bipolare Prüfspannung}

Im Folgenden werden die Ergebnisse für eine bipolare Impulsspannung mit einem Scheitelwert von $1090 \mathrm{~V}$ betrachtet. Die zeitlichen Verläufe der Gesamtspannung und des berechneten Stromverlaufs sind in Abb. 14 (a) zu sehen. Die Gesamtspannung teilt sich auf die Spannungen über Dielektrikum und Gasraum auf, wobei das Verhältnis der beiden Spannungen durch den Entladeprozess verändert wird. Der Stromverlauf setzt sich aus dem berechneten Kompen-

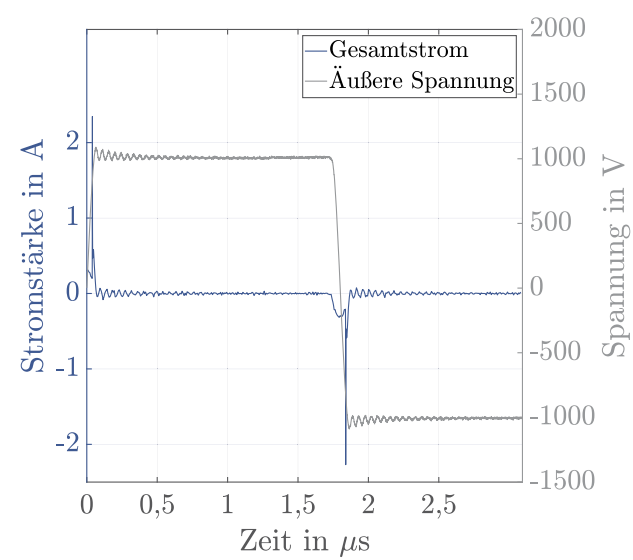

(a) Spannungs- und Stromverlauf.

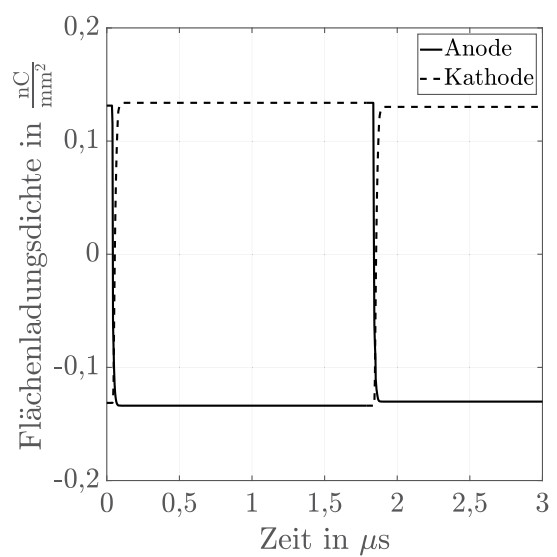

(b) Verlauf der Oberflächenladung.

Abb. 14. Zeitlicher Verlauf der berechneten Ladungsträgerstroms und der externen Spannung sowie der Oberflächenladungen für bipolare Prüfspannungen

sationsstrom und dem kapazitiven Verschiebungsstrom zusammen. Letzterer ergibt sich aus dem Zusammenhang aus der angelegten Prüfspannung und der Kapazität des Prüflings und wird mittels Messung am Oszilloskop ermittelt. Die abgebildeten Ergebnisse zeigen einen stationären Zustand, bei dem an jeder Pulsflanke eine Entladung mit signifikantem Strombeitrag auftritt. Entsprechend wechselt die Flächenladungsdichte an Anode und Kathode ihr Vorzeichen während des Entladevorgangs. Wegen des Polaritätswechsels ist der Verlauf der Flächenladungsdichte unstetig. Es ist zu erwähnen, dass die erste Entladung, die in Abb. 14 (a) nicht zu sehen ist, keinen signifikanten Strombeitrag liefert und somit nicht als Teilentladung zu erkennen ist. Allerdings wird durch diese Entladung eine Oberflächenladung generiert, die im Moment des Polaritätswechsels die Spannung im Gasraum erhöht und die Zündbedingung somit stärker überschritten ist. Dadurch wird beim ersten Polaritätswechsel der Spannung ein signifikanter Strombeitrag erreicht. Anschließend stellt sich nach wenigen Polaritätswechseln der gleichgewichtige Zustand in Abb. 14 ein, bei dem der Betrag der Flächenladungsdichten nur noch im Bereich weniger Prozent variiert und die Stromamplituden der Entladungen annähernd gleich sind.

Die Teilentladungseinsetzspannung ist der Wert der Prüfspannung, ab dem Entladepulse mit einem signifikanten Strombeitrag auftreten und somit im Verschiebungsstrom sichtbar sind. Die vorge- 


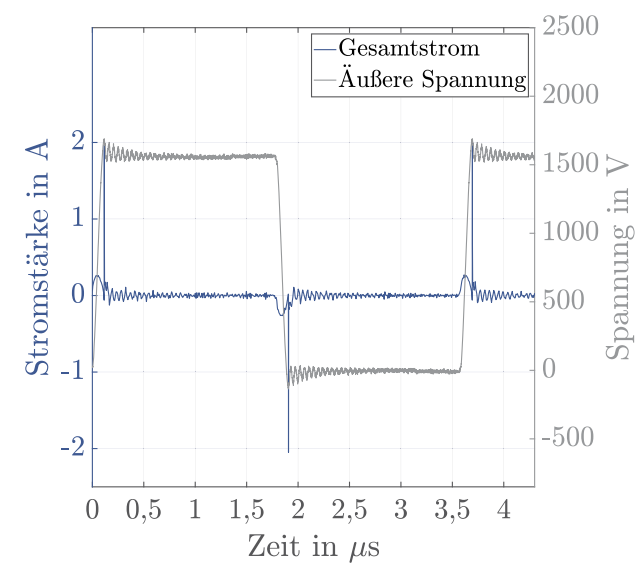

(a) Spannungs- und Stromverlauf.

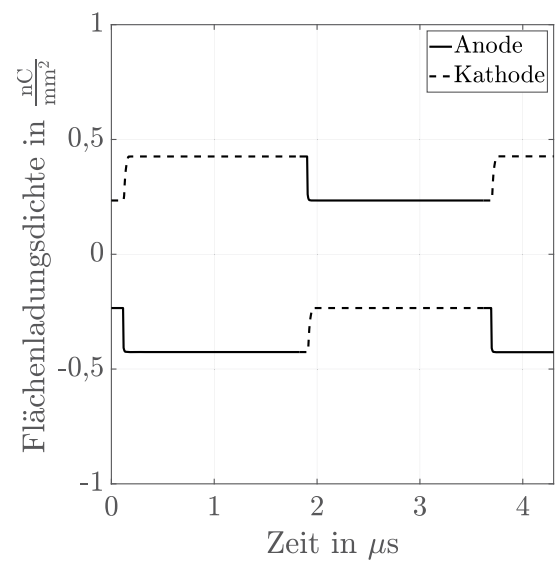

(b) Verlauf der Oberflächenladung.

Abb. 15. Zeitlicher Verlauf der berechneten Ladungsträgerstroms und der externen Spannung sowie der Oberflächenladungen für unipolare Prüfspannungen

stellten Ergebnisse beziehen sich auf den Scheitelwert $U_{p}$ der Spannung. Aus den Berechnungen ergibt sich, dass die PDIV für bipolare Spannungen und die verwendeten Eingangsparameter in etwa bei 1090 V liegt. Die simulierte PDIV liegt somit 50 V oberhalb der kritischen Spannung. Dies ist auf den Verlauf der Prüfspannung und auf das Überschwingverhalten zurückzuführen. Der kurze Zeitbereich des Überschwingens reicht nicht aus, um eine selbstständige Entladung voranzutreiben. Unterhalb einer Spannung von $1090 \mathrm{~V}$ entstehen keine Entladepulse und es fließt neben dem Verschiebungsstrom nur ein kleiner lonisierungsstrom. Die Simulationsergebnisse zeigen, dass die Spannung, ab der regelmäßig Teilentladungen auftreten, (engl.: Repetitive Partial Discharge Inception Voltage, kurz: RPDIV) bei bipolaren Prüfspannungen der PDIV entspricht. Dieser Zusammenhang begründet sich durch die Ladungsträger auf den dielektrischen Oberflächen. Diese lagern sich bereits bei der ersten Entladung an den Barrieren an und verstärken bei jedem Polaritätswechsel das Feld im Luftraum, wodurch weitere Teilentladungen begünstigt werden.

\subsubsection{Unipolare Prüfspannung}

Analog zu den Ergebnissen für eine bipolare Testspannung tritt beim ersten unipolaren Spannungspuls eine Entladung mit sehr geringer Stromamplitude (im Stromverlauf nicht sichtbar) auf, die zu ei-

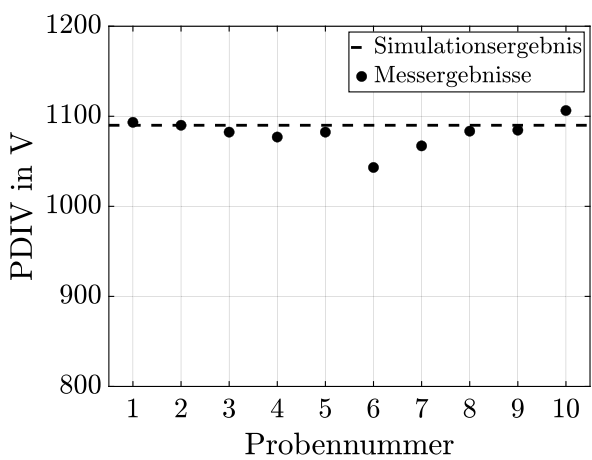

Abb. 16. Vergleich zwischen Mess- und Simulationsergebnissen für bipolare Impulsspannung

ner Ansammlung einer Oberflächenladung führt. Da kein Polaritätswechsel stattfindet, kommt es bei einem Scheitelwert von $1090 \mathrm{~V}$ nicht zu einer Teilentladung. Während der zweiten Hälfte der Periodendauer, in der die externe Spannung 0 V beträgt, ist die Spannung über dem Gasraum bedingt durch die Flächenladungen negativ. Wird die Spannung weiter erhöht, treten vereinzelt Teilentladungen auf. Ab einer Spannung von $1690 \mathrm{~V}$ treten auch im Bereich der fallenden Flanke der Pulsspannung Teilentladungen entgegengesetzter Polarität auf, wie in Abb. 15a zu erkennen ist. Durch die Teilentladungen während der ersten Hälfte der Periodendauer bildet sich eine ausreichend große Oberflächenladung, um im Bereich der fallenden Flanke Teilentladungen entgegengesetzter Polarität auszulösen. Die Erkenntnis, dass bei unipolarer Impulsspannung auch im Bereich der fallenden Flanke TE auftreten können, deckt sich mit Messergebnissen aus der Literatur [25]. Es bildet sich für den zeitlichen Verlauf der Oberflächenladungen ein gleichgewichtiger Zustand aus, wie in Abb. 15 (b) gezeigt wird.

\subsection{Messergebnisse}

Zur Verifikation der Simulationsergebnisse wird die PDIV für zehn Prüflinge bei bipolarer Impulsspannung ermittelt. Darüber hinaus wird der durch die Simulation berechnete Strompuls mit dem gemessenen Signal bei einer Teilentladung verglichen. Für unipolare Impulsspannung können keine TE-Messungen durchgeführt werden, da vor Überschreiten der Sperrspannung der Halbleiter von $1200 \mathrm{~V}$ keine wiederkehrenden TE aufgetreten sind. Die gemessenen Werte für die PDIV beziehen sich auf den Scheitelwert $U_{p}$ der angelegten Prüfspannung. Durch den Vergleich der Mess- mit den Simulationsergebnissen in Abb. 16 wird deutlich, dass eine gute Übereinstimmung mit den gemessenen Werten erreicht wird. Der simulierte Wert von $1090 \mathrm{~V}$ liegt zwischen dem gemessenen Minimum von $1043 \mathrm{~V}$ und dem Maximum von $1106 \mathrm{~V}$.

In Abb. 17 werden die simulierten und gemessenen TE-Impulse verglichen. Der Vergleich lässt erkennen, dass der Scheitelwert von 2,35 A des simulierten Stroms in derselben Größenordnung des gemessenen Stroms von 2,16 A liegt. Das zeitliche Auftreten liegt jeweils im Bereich des ersten Maximums der Spannung. Die gemessenen, starken Oszillationen im Stromverlauf haben ihren Ursprung aus Resonanzen im Prüfkreis. Der hier betrachtete Messaufbau besteht aus dem untersuchten Drilleiter-Prüfling, Zuleitungen und einer leistungselektronischen Schaltung zur Bereitstellung der Impulsspannung. Diese Komponenten besitzen unterschiedliche resistive, kapazitive und induktive Leitungsbeläge. Durch den Teilentladungsimpuls und den damit verbundenen schnellen Stromanstieg entsteht eine Rückwirkung auf den Spannungsverlauf. Das hat zur Folge, dass ein verstärktes Schwingungsverhalten der Spannung 


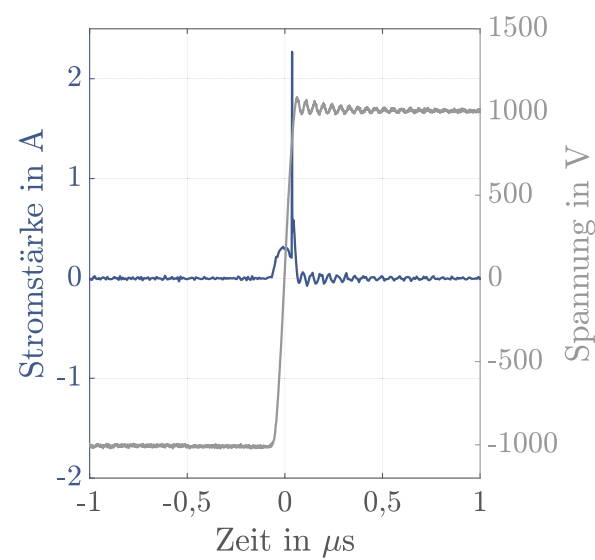

(a) Simlierter Spannungs- und Stromverlauf.

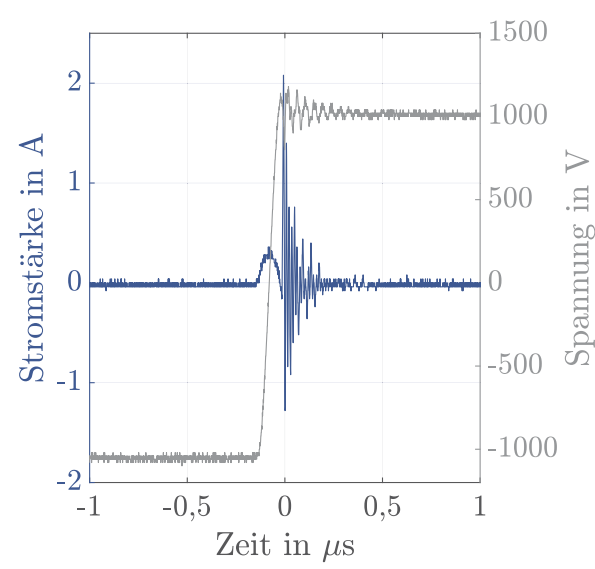

(b) Gemessener Spannungs- und Stromverlauf.

Abb. 17. Zeitlicher Verlauf der simulierten und gemessenen Spannungs- und Stromverläufe

auftritt und der Spannungsverlauf durch ein hochfrequentes Überschwingen überlagert wird. Diese Effekte werden in der Simulation nicht abgebildet. Durch Integration des Stromverlaufs des TEImpulses kann die freigesetzte Ladung bestimmt werden. Es ist zu erkennen, dass die freigesetzte Ladung nach Abklingen der Oszillation für beide Verläufe in derselben Größenordnung liegen. Der vergleichende zeitliche Verlauf der simulierten und gemessenen Ladung ist in Abb. 18 dargestellt. Der erreichte Endwert der simulierten und gemessenen Ladung beträgt circa 11,3 nC bzw. 12,5 nC.

\section{Schlussfolgerung}

In dieser Arbeit wird ein ladungsträgerbasierter Ansatz zur Modellierung von Teilentladungsprozessen vorgestellt. Zunächst wird das Laplace-Feld der Anordnung mit einem Finite-Elemente-Modell berechnet. Anschließend wird mithilfe des Townsend-Kriteriums die kritische elektrische Feldlinie bestimmt, um die sich mit hoher Wahrscheinlichkeit der Entladekanal ausbreiten wird. Durch Lösung der Fluss-Kontinuitätsgleichungen werden der Stromimpuls und die Oberflächenladungen berechnet. Dieser Ansatz erlaubt es, die Teilentladungsprozesse für unipolare und bipolare Impulsspannungen zu charakterisieren. Die Berechnung der Entladestromstärke erlaubt Rückschlüsse auf die Intensität einer Teilentladung. Es soll somit möglich sein, detailliertere Modelle des Alterungsverhaltens der Isoliermaterialien zu erstellen. Die Validierung des Modells findet durch

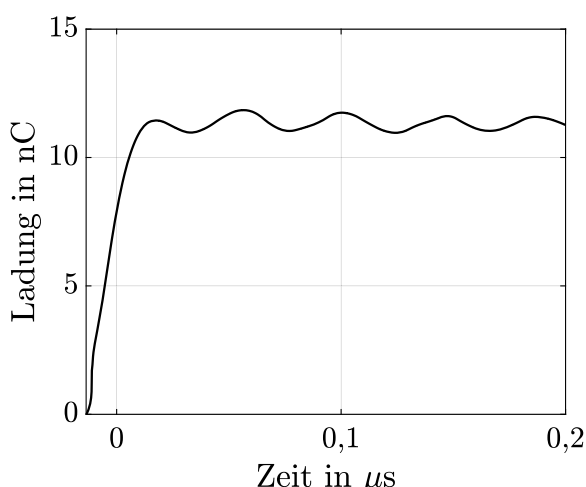

(a) Simlierter Verlauf der Ladung.

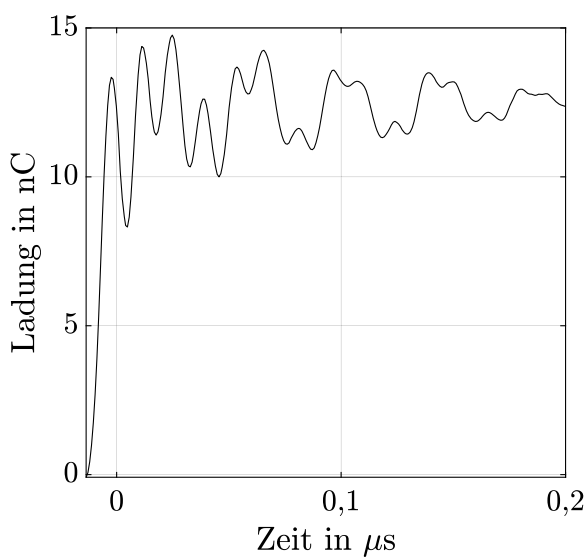

(b) Gemessener Verlauf der Ladung.

Abb. 18. Zeitlicher Verlauf der bei einer TE entstehenden simulierten und gemessenen Ladung

Teilentladungsmessungen an Twisted-Pairs statt. Durch Vergleich der Mess- und Simulationsergebnisse wird deutlich, dass sich das Modell sowohl zur qualitativen als auch zur quantitativen Betrachtung von Ladungsträgerphänomenen eignet. Es können für bipolare Rechteckspannungen sowohl die TE-Einsetzspannung als auch der zeitabhängige Stromverlauf der Entladeanordnung modelliert werden.

Funding Note Open Access funding enabled and organized by Projekt DEAL.

Hinweis des Verlags Der Verlag bleibt in Hinblick auf geografische Zuordnungen und Gebietsbezeichnungen in veröffentlichten Karten und Institutsadressen neutral.

Open Access Dieser Artikel wird unter der Creative Commons Namensnennung 4.0 International Lizenz veröffentlicht, welche die Nutzung, Vervielfältigung, Bearbeitung, Verbreitung und Wiedergabe in jeglichem Medium und Format erlaubt, sofern Sie den/die ursprünglichen Autor(en) und die Quelle ordnungsgemäß nennen, einen Link zur Creative Commons Lizenz beifügen und angeben, ob Änderungen vorgenommen wurden. Die in diesem Artikel enthaltenen Bilder und sonstiges Drittmaterial unterliegen ebenfalls der genannten Creative Commons Lizenz, sofern sich aus der Abbildungslegende nichts anderes ergibt. Sofern das betreffende Material nicht unter der genannten Creative Commons Lizenz steht und die betreffende Handlung nicht nach gesetzlichen Vorschriften erlaubt ist, ist für die oben aufgeführten Weiterverwendungen des Materials die Einwilligung des jeweiligen Rechteinhabers einzuholen. Weitere Details zur Lizenz entnehmen Sie bitte der Lizenzinformation auf http://creativecommons.org/licenses/by/4.0/deed.de. 


\section{Literatur}

1. Kuffel, E., Zaengl, W. S., Kuffel, J. (2000): High voltage engineering - fundamentals. Oxford: Butterworth-Heinemann. Imprint Newnes

2. Pauli, F., Yang, L., Schröder, M., Hameyer, K. (2019): Lebensdauerabschätzung von Wicklungsisolierstoffsystemen in SiC-betriebenen elektrischen Niederspannungsmaschinen. E\&l, Elektrotech. Inf.tech., 136(2), 175-183.

3. Pauli, F., Driendl, N., Hameyer, K. (2019): Study on temperature dependence of partial discharge in low voltage traction drives. In 2019 IEEE workshop on electrical machines design, control and diagnosis (WEMDCD) (Bd. 1, S. 209-214).

4. Jung, C. (2017): Power up with 800-V systems: the benefits of upgrading voltage power for battery-electric passenger vehicles. IEEE Electrif. Mag., 5, 53-58.

5. Fabiani, D. (2003): Accelerated degradation of ac-motor winding insulation due to voltage waveforms generated by adjustable speed drives. Dissertation, University of Bologna.

6. Florkowska, B., Zydron, P., Florkowski, M. (2011): Effects of inverter pulses on the electrical insulation system of motors. New York: IEEE Press.

7. Ruf, A., Paustenbach, J., Franck, D., Hameyer, K. (2017): A methodology to identify electrical ageing of winding insulation systems. In International electric machines and drives conference, IEMDC 2017

8. Kaufhold, M., Borner, G., Eberhardt, M., Speck, J. (1996): Failure mechanism of the interturn insulation of low voltage electric machines fed by pulse-controlled inverters. IEEE Electr. Insul. Mag., 12(5), 9-16.

9. Madonna, V., Giangrande, P., Lusuardi, L., Cavallini, A., Gerada, C., Galea, M. (2019): Thermal overload and insulation aging of short duty cycle, aerospace motors. IEEE Trans. Ind. Electron., 67, 2618-2629.

10. Hayakawa, N., Okubo, H. (2005): Partial discharge characteristics of inverter-fed motor coil samples under ac and surge voltage conditions, S. 5-10.

11. Hikita, M., Yanaze, N., Uchimura, T., Kozako, M., Tomizawa, K., Ohya, M. (2016): Partial discharge and breakdown characteristics of inverter-fed motor winding with micro cellular coating. In 2016 IEEE conference on electrical insulation and dielectric phenomena (CEIDP), Toronto, ON (S. 482-485).

12. Küchler, A. (2017): Hochspannungstechnik Grundlagen - Technologie - Anwendungen. 4. Aufl. Berlin: Springer.

13. Norm DIN EN 60172 (2016): Prüfverfahren zur Bestimmung des Temperaturindex von Lackdrähten und bandumwickelten Drähten. IEC 60172 2015. Deutsche Fassung EN 60172:2015
14. Morrow, R. (1985): Theory of negative corona in oxygen. Phys. Rev. A, 32, 1799-1809.

15. Braun, D., Kuechler, U., Pietsch, G. (1991): Microdischarges in air-fed ozonizers. J. Phys. D, Appl. Phys., 24(4), 564-572.

16. Steinle, P., Morrow, R., Roberts, A. J. (1989): Use of implicit and explicit flux-corrected transport algorithms in gas discharge problems involving non-uniform velocity fields. J. Comput. Phys., 85(2), 493-499.

17. Boris, J. P., Book, D. L. (1976): Flux-corrected transport. III. Minimal-error FCT algorithms. J. Comput. Phys., 20(4), 397-431.

18. Kang, W. S., Park, J. M., Kim, Y., Hong, S. H. (2003): Numerical study on influences of barrier arrangements on dielectric barrier discharge characteristics. IEEE Trans. Plasma Sci., 31(4), 504-510.

19. Humpert, C. (2002): Modellierung des dynamischen Verhaltens von OberflächenBarrierenentladungen und der Ozonerzeugung. Dissertation, RWTH Aachen.

20. Golubovskii, Yu. B., Maiorov, V. A., Behnke, J., Behnke, J. F. (2002): Influence of interaction between charged particles and dielectric surface over a homogeneous barrier discharge in nitrogen. J. Phys. D, Appl. Phys., 35(8), 751-761.

21. Braun, D. (1990): Zum Wirkungsgrad von Ozonerzeugern. RWTH Aachen, Dissertati-

22. Norm DIN EN 60034-18-41 (November 2014): Drehende elektrische Maschinen Teil 18-41: Qualifizierung und Qualitätsprüfungen für teilentladungsfreie elektrische Isoliersysteme (Typ 1) in drehenden elektrischen Maschinen, die von Spannungsumrichtern gespeist werden.

23. Pauli, F., Ruf, A., Hameyer, K. (2020): Low voltage winding insulation systems under the influence of high du/dt slew rate inverter voltage. Arch. Elektrotech., 69(1), $187-202$

24. Norm DIN EN 60317-0-1 (September 2014): Technische Lieferbedingungen für bestimmte Typen von Wickeldrähten - Teil 0-1: Allgemeine Anforderungen - Runddrähte aus Kupfer, lackisoliert.

25. Fabiani, D., Montanari, G. C., Cavallini, A., Mazzanti, G. (2004): Relation between space charge accumulation and partial discharge activity in enameled wires under PWM-like voltage waveforms. In IEEE transactions on dielectrics and electrical insulation (Bd. 11, S. 393-405), Nr. 3

26. Hoang, A. T., Serdyuk, Y. V. Gubanski, S. M. (2018): Charge transport in a doublelayered enamel insulation during surface potential decay. In IEEE transactions on dielectrics and electrical insulation (Vol. 25, S. 702-712), Nr. 2.

\section{Anhang}

Tab. 2. Eingangsparameter des Simulationsmodells

\begin{tabular}{|c|c|}
\hline Parameter & Bezeichnung/Wert \\
\hline Verwendeter Runddraht & $\begin{array}{l}\text { Dahréntråd DAMID } 200 \\
\text { Grad } 2\end{array}$ \\
\hline Leiterradius $r_{\text {Leiter }}$ & $0,5 \mathrm{~mm}$ \\
\hline Grundlack & $\begin{array}{l}\text { THEIC-mod. Polyester } \\
\text { oder Polyesterimid }\end{array}$ \\
\hline Überzuglack & Polyamidimid \\
\hline Gesamtisolierdicke $d_{\text {Iso }}$ & $39,25 \mu \mathrm{m}$ \\
\hline Innere Isolierdicke & $19,5 \mu \mathrm{m}$ \\
\hline Dielektrizitätszahl PEI & $3,5[26]$ \\
\hline Dielektrizitätszahl PAI & $4,0[26]$ \\
\hline Minimaler & $2 \mu \mathrm{m}$ \\
\hline \multicolumn{2}{|l|}{ Drahtabstand $d_{\min }$} \\
\hline Länge der & $125 \mathrm{~mm}$ \\
\hline \multicolumn{2}{|l|}{ Verdrillung / Pruefling } \\
\hline $\begin{array}{l}\text { Umgebungstempera- } \\
\operatorname{tur} \theta\end{array}$ & $20^{\circ} \mathrm{C}$ \\
\hline Gasdruck $p$ & $1013,25 \mathrm{hPa}$ \\
\hline Neutrale & $24,7 \cdot 10^{24} \mathrm{~m}^{-3}$ \\
\hline \multicolumn{2}{|l|}{ Teilchendichte $n$} \\
\hline Sekundärkoeffizient $\gamma_{i o n}$ & $0,8 \cdot 10^{-3}$ \\
\hline Sekundärkoeffizient $\gamma_{\mathrm{ph}}$ & $2,5 \cdot 10^{-3}$ \\
\hline $\begin{array}{l}\text { Elektronenflucht- } \\
\text { frequenz } f_{\text {esc }}\end{array}$ & $4 \cdot 10^{15} \mathrm{~s}^{-1}$ \\
\hline Adsorptionsenergie $\Phi_{\sigma}$ & $0.9 \mathrm{eV}$ \\
\hline Fremdionisationsrate & $100 \mathrm{~s}^{-1} \mathrm{~cm}^{-3}$ \\
\hline Prüflingskapazität $C_{\text {ges }}$ & $13,3 \mathrm{pF}$ \\
\hline
\end{tabular}




\section{Autoren}

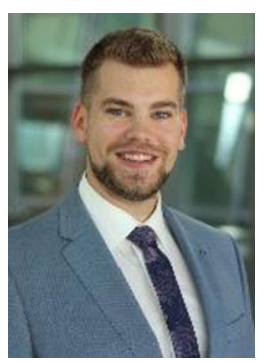

\section{Niklas Driendl}

schloss im Oktober 2018 das ElektrotechnikStudium an der RWTH Aachen mit dem Master of Science ab. Seit Januar 2019 arbeitet er als wissenschaftlicher Mitarbeiter am Institut für Elektrische Maschinen der RWTH Aachen. Seine Forschungsschwerpunkte liegen im Bereich der Auslegung von Isoliersystemen elektrischer Niederspannungsmaschinen und der Untersuchung von Teilentladungsphänomenen.

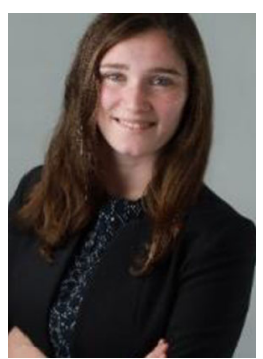

\section{Clara lütke Jüdefeld}

studierte Wirtschaftsingenieurwesen mit Fachrichtung Elektrische Energietechnik an der RWTH Aachen und schloss im Juni 2020 mit dem Master of Science ab. Während des Studiums arbeitete sie als studentische Hilfskraft und nach ihrem Abschluss als wissenschaftliche Hilfskraft am Institut für Elektrische Maschinen der RWTH Aachen. Ihr Forschungsinteresse fokussiert sich auf die Auslegung von Niederspannungsmaschinen und deren Isoliersystemen.

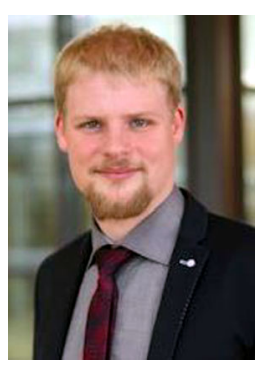

\section{Florian Pauli}

studierte Elektrotechnik an der RWTH Aachen und schloss im April 2017 mit dem Master of Science ab. Seitdem arbeitet er als wissenschaftlicher Mitarbeiter am Institut für Elektrische Maschinen der RWTH Aachen. Seine Forschungsschwerpunkte liegen im Bereich der Analyse, Charakterisierung und Auslegung von Isoliersystemen elektrischer Niederspannungsmaschinen.

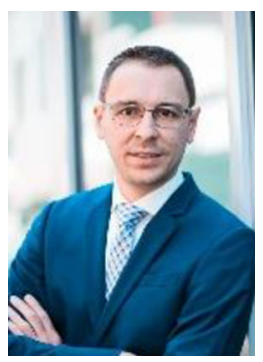

Michael Schröder

studierte an der RWTH Aachen Elektrotechnik und schloss im Mai 2012 mit dem Diplom ab. Im Anschluss wurde er wissenschaftlicher Mitarbeiter am Institut für Elektrische Maschinen der RWTH Aachen. Von Oktober 2014 bis Oktober 2017 war er Gruppenleiter der Gruppe "Analyse und Design" mit Forschungsthemen im Bereich der weich-und hartmagnetischen Materialien, Isolierstoffsysteme sowie dem strukturdynamischen und akustischen Verhalten elektrischer Maschinen. Seit November 2017 ist er ein Oberingenieur des Instituts. Im Februar 2020 promovierte Dr. Schröder zum Thema "Modellbildung und Analyse von Kraftanregungen in permanentmagneterregten elektrischen Maschinen unter Berücksichtigung von Fertigungseinflüssen".

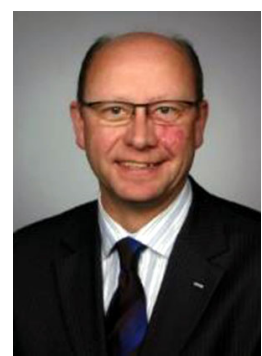

Kay Hameyer

erwarb sein Diplom in Elektrotechnik von der Universität Hannover und promovierte an der Technischen Universität Berlin. Nach seinem Studium arbeitete er für die Robert Bosch GmbH in Stuttgart als Entwicklungsingenieur für permanentmagneterregte Servomotoren und Kraftfahrzeugbordnetzkomponenten. Bis 2004 war Dr. Hameyer Professor für Numerische Feldberechnungen und Elektrische Maschinen an der KU Leuven in Belgien. Seit 2004 ist er Professor und Leiter des Instituts für Elektrische Maschinen der RWTH Aachen. 2006 war er Vizedekan der Fakultät für Elektrotechnik und Informationstechnik der RWTH Aachen und von 2007 bis 2009 Dekan der Fakultät. Seine Forschungsschwerpunkte liegen im Bereich der numerischen Feldberechnung und -optimierung, dem Entwurf und der Regelung elektrischer Maschinen, insbesondere von permanentmagneterregten Maschinen und Drehfeldmaschinen sowie der Entwicklung von Methoden für die virtuelle Realität. Seit vielen Jahren beschäftigt sich die Arbeit von Dr. Hameyer mit der Magnetschwebetechnik für Antriebssysteme, der elektromagnetischen Geräuschanregung elektrischer Maschinen und der Charakterisierung ferromagnetischer Materialien und Isolierwerkstoffe. Er ist Autor von mehr als 350 Journal-Publikationen, mehr als 700 internationalen Konferenzveröffentlichungen und Autor von vier Büchern. Dr. Hameyer ist Mitglied im VDE, IEEE Senior Member und Fellow des IET. 